\title{
NIH push for women's health
}

Heart disease, osteoporosis and cancer win a new claim on NIH's attention as political pressure in the United States drives women's issues to the top of the research agenda.

FOR a year now, the US National Institutes of Health (NIH) have had an Office of Research on Women's Health. When cardiologist Bernadine Healy became the first woman head of NIH earlier this year, she was quick to announce a major research initiative on women's health. Whatever her difficulties with congressman John Dingell over the NIH's fraud office, Healy will win universal praise from Congress for this politically correct stance on women. It is an interesting development.

A comerstone of the initiative will be a $\$ 500$ million decade-long study of between 60,000 and 70,000 post-menopausal women who, by virtue of age, are at risk of having a heart attack, dying of cancer or becoming disabled by aged and brittle bones.

The study is being designed to assess the effects on women's health of diet, hormone-replacement therapy, calcium and vitamin D supplements, and exercise. The study will probably also cover the use of anti-oxidants, the relative value of detecting disease early and the prevention of smoking. It will be the largest epidemiological analysis of its kind ever undertaken in the United States.

Healy says this study of post-menopausal women will be but one of several initiatives coordinated through the Office of Research on Women's Health, which was created, before she became director, at the insistence of the Women's Health Caucus of the Congress, urged on by a group of women in science who have since formed the Society for the Advancement of Women's Health Research.

The premise underlying the movement is that research on men's health cannot be readily extended to women, and that research on women of one ethnic group cannot necessarily be applied to another. "Hence, research must take into account the cultural differences that occur among such diverse groups as African American, Hispanic, Native American and Asian women." The women's movement also places a good deal of faith in the belief that disease can be prevented by appropriate behaviour, implying that the behavioural and social sciences are as important as the traditional biomedical sciences in creating a new research agenda.

Women's health did not spring onto the political agenda just in the past year. The issue has been gaining momentum since the early 1980 s, when the government appointed a task force whose recommendations, not surprisingly, sound very much like those being made now. In response to previous calls for more research on women, NIH made an inventory of the research they supported in 1987 , calculating that 13.5 per cent of their funds, or $\$ 778$ million, were spent on problems unique to women, while approximately 80 per cent went to basic research. That leaves less than 7 per cent for research on disorders unique to men.

Nevertheless, a case can be made that certain types of research have focused almost exclusively on men to the detriment of women. Heart disease is one. In the United States, more than 750,000 people a year die of heart disease - and 49 per cent are women. Yet the general impression is that heart disease is a killer of men, largely because men have heart attacks in middle age while most women are immune until they pass menopause. So the major clinical trials of beta-blockers, lipid-lowering agents, angioplasty and bypass surgery have been carried out almost exclusively with men.

Moreover, studies published in the $\mathrm{New}$ England Journal of Medicine (266, 221230; 25 July 1991) show that physicians tend to treat heart disease in women less aggressively than in men unless or until a woman has an outright heart attack. Healy calls this the 'Yentl Syndrome' after the woman in Isaac Bashevis Singer's short story who had to dress up as a man in order to study the Talmud.

As a result, there are demands not only that women should routinely be included in clinical trials but also that gender differences be taken into account in designing protocols. A simple example is that of oestrogen therapy, now known to reduce the risk of heart disease in post-menopausal women by as much as 47 per cent. But an oestrogen trial 20 years ago yielded no evidence of benefit. (It was conducted in middle-aged men.) Now, the NIH have rewritten grant application forms, requiring that clinical trials include "an adequate number of women" unless the investigator provides a reason why there should not be that will pass muster with reviewers. (The Institute of Medicine has been asked to advise the NIH on inclusion or exclusion of women of child-bearing age.)

NIH's new-found emphasis on women is just one more example of how the institutes have for decades been pushed (or led, depending upon point of view) by political pressure. Special initiatives satisfy political customers and are, as often as not, motivated as much by the desire to find a cure as by scientific opportunity. There are elements of both in the women's health initiative.

A recent three-day NIH workshop, convened to plan a research agenda for a decade and more, tells the tale. Very little was said about scientific opportunity but there was a lot of talk about the social and medical problems perceived to be particular to women. The meeting, intended to produce a genuine research plan, not surprisingly produced a laundry list of needs but no corresponding list of research projects that might yield solutions.

The first draft report of the proceedings (which needs further iteration) lists every conceivable aspect of a woman's life, from conception to death eight or nine decades later, as urgent matters for NIH research. It calls for research on the causes of teenage pregnancy, the effects of poverty on infants and the "dynamics that support a woman's decision to breastfeed". Depression, suicide and post-traumatic stress disorder in teenage girls are on the list, as are the increasing incidence of breast cancer in adult women and postmenopausal coronary disease.

Although light on proposals for research projects, the draft is replete with exhortations to change behaviour to prevent disease, sexually transmitted and cardiovascular alike. There is even a proposal to study ways of persuading young women to exercise so as to develop strong bones to ward off osteoporosis later in life. (It may be the first time NIH have been asked to get girls to take gym.)

It is vital that the relationship between behaviour and disease should be rigorously dissected to separate fad from wishful thinking from valid science. Many people, including an apparent majority at the women's health conference, place as much emphasis on behaviour as on medicine, a view that would predicate a change in the way NIH see their mission. This is entirely appropriate, provided it is accompanied by hard-eyed protocols for research in behavioural science that would advance the field.

What is missing from the draft are proposals for scientifically innovative ideas. The plan in Congress is to bring the National Institute of Mental Health back within the NIH fold to strengthen that tendency. But that will be good for women only if it is accompanied by first-rate science. That is the challenge. Barbara J. Culliton 